\title{
Some Remarkable Identities Involving Numbers
}

\author{
Rafał Ziobro \\ Department of Carbohydrate Technology \\ University of Agriculture \\ Krakow, Poland
}

\begin{abstract}
Summary. The article focuses on simple identities found for binomials, their divisibility, and basic inequalities. A general formula allowing factorization of the sum of like powers is introduced and used to prove elementary theorems for natural numbers.

Formulas for short multiplication are sometimes referred in English or French as remarkable identities. The same formulas could be found in works concerning polynomial factorization, where there exists no single term for various identities. Their usability is not questionable, and they have been successfully utilized since for ages. For example, in his books published in 1731 (p. 385), Edward Hatton [3] wrote: "Note, that the differences of any two like powers of two quantities, will always be divided by the difference of the quantities without any remainer...".

Despite of its conceptual simplicity, the problem of factorization of sums/differences of two like powers could still be analyzed [7, giving new and possibly interesting results [6].
\end{abstract}

MSC: 11A67 03B35

Keywords: identity; divisibility; inequations; powers

MML identifier: NEWTON01, version: 8.1.03 5.26.1224

The notation and terminology used in this paper have been introduced in the following articles: [8], [9], [5], [4], and [2].

From now on $a, b, c, d, x, j, k, l, m, n$ denote natural numbers, $p, q, t, z, u$, $v$ denote integers, and $a_{1}, b_{1}, c_{1}, d_{1}$ denote complex numbers.

Let $u, v$ be even integers. One can check that $u-v$ is even.

Let $u$ be an odd integer. Let us consider $k$. Observe that $u^{k}$ is odd.

Let $k$ be a positive natural number and $u$ be an even integer. Let us observe that $u^{k}$ is even. 
Now we state the propositions:

(1) $a_{1}^{2}-b_{1}^{2}=\left(a_{1}-b_{1}\right) \cdot\left(a_{1}+b_{1}\right)$.

(2) $\left(2 \cdot a_{1}+1\right)^{2}+\left(2 \cdot a_{1}^{2}+2 \cdot a_{1}\right)^{2}=\left(2 \cdot a_{1}^{2}+2 \cdot a_{1}+1\right)^{2}$.

(3) $a_{1}^{2}+a_{1} \cdot b_{1}+b_{1}^{2}=\frac{3 \cdot\left(a_{1}+b_{1}\right)^{2}+\left(a_{1}-b_{1}\right)^{2}}{4}$.

(4) If $a$ is odd, then there exists $b$ such that $a^{2}+b^{2}=(b+1)^{2}$. The theorem is a consequence of (2).

(5) $\frac{\left(a_{1}{ }^{m}+b_{1}{ }^{m}\right) \cdot\left(a_{1}{ }^{n}-b_{1}{ }^{n}\right)+\left(a_{1}{ }^{n}+b_{1}{ }^{n}\right) \cdot\left(a_{1}{ }^{m}-b_{1}{ }^{m}\right)}{2}=a_{1}{ }^{m+n}-b_{1}{ }^{m+n}$.

(6) If $a^{m}+b^{m} \leqslant c^{m}$, then $a \leqslant c$.

(7) Suppose $\left(a_{1}+b_{1}\right)^{n+1}=a_{1}{ }^{n+1}+b_{1}{ }^{n+1}+a_{1} \cdot b_{1} \cdot c_{1}$. Then $\left(a_{1}+b_{1}\right)^{n+2}=$ $a_{1}{ }^{n+2}+b_{1}{ }^{n+2}+a_{1} \cdot b_{1} \cdot\left(a_{1}{ }^{n}+b_{1}{ }^{n}+c_{1} \cdot\left(a_{1}+b_{1}\right)\right)$.

(8) $\frac{\left(a_{1}{ }^{m}+b_{1}{ }^{m}\right) \cdot\left(a_{1}{ }^{n}+b_{1}{ }^{n}\right)+\left(a_{1}{ }^{n}-b_{1}{ }^{n}\right) \cdot\left(a_{1}{ }^{m}-b_{1}{ }^{m}\right)}{2}=a_{1}{ }^{m+n}+b_{1}{ }^{m+n}$.

(9) $a_{1}{ }^{m+1}+b_{1}{ }^{m+1}=\frac{\left(a_{1}{ }^{m}+b_{1}{ }^{m}\right) \cdot\left(a_{1}+b_{1}\right)+\left(a_{1}-b_{1}\right) \cdot\left(a_{1}{ }^{m}-b_{1}{ }^{m}\right)}{2}$. The theorem is a consequence of (8).

(10) $(a-b) \cdot\left(a^{m}-b^{m}\right) \geqslant 0$.

(11) $a^{m+1}+b^{m+1} \geqslant \frac{\left(a^{m}+b^{m}\right) \cdot(a+b)}{2}$. The theorem is a consequence of (9) and (10).

(12) If $a^{m}+b^{m} \leqslant c^{m}$, then there exists $x$ such that $a^{m}+b^{m} \leqslant(a+x)^{m}$. The theorem is a consequence of (6).

(13) $a_{1}{ }^{m+1}-b_{1}{ }^{m+1}=\frac{\left(a_{1}{ }^{m}+b_{1}{ }^{m}\right) \cdot\left(a_{1}-b_{1}\right)+\left(a_{1}+b_{1}\right) \cdot\left(a_{1}{ }^{m}-b_{1}{ }^{m}\right)}{2}$. The theorem is a consequence of (5).

(14) $\quad a^{m+1}-b^{m+1}=\frac{(a-b) \cdot\left(t \cdot(a+b)+a^{m}+b^{m}\right)}{2}$ if and only if $a^{m}-b^{m}=(a-b) \cdot t$.

(15) $\left(\frac{c_{1}{ }^{n}}{2}+\frac{c_{1}}{2}\right)^{2}-\left(\frac{c_{1}{ }^{n}}{2}-\frac{c_{1}}{2}\right)^{2}=c_{1}{ }^{n+1}$.

(16) $a^{3}-b^{3}=\frac{(a-b) \cdot\left((a+b) \cdot(a+b)+a^{2}+b^{2}\right)}{2}$. The theorem is a consequence of (1) and (14).

(17) If $c^{m} \geqslant a^{m}+b^{m}$ and $a>0$ and $b>0$, then $c^{m+1}>a^{m+1}+b^{m+1}$. The theorem is a consequence of (6).

(18) If $c^{m} \geqslant a^{m}+b^{m}$ and $a>0$ and $b>0$ and $k>0$, then $c^{k+m}>$ $a^{k+m}+b^{k+m}$.

Proof: Define $\mathcal{P}$ [natural number] $\equiv c^{\$_{1}+m+1}>a^{\$_{1}+m+1}+b^{\$_{1}+m+1}$ and $a>0$ and $b>0$. $\mathcal{P}[0]$. If $\mathcal{P}[x]$, then $\mathcal{P}[x+1]$. For every $j, \mathcal{P}[j]$ from [1, Sch. 2].

(19) If $c^{m} \geqslant a^{m}+b^{m}$, then $c^{k+m} \geqslant a^{k+m}+b^{k+m}$. The theorem is a consequence of (18).

(20) If $c^{n}>a^{n}+b^{n}$, then $c^{k+n}>a^{k+n}+b^{k+n}$.

Proof: Consider $m$ such that $n=1+m$. Define $\mathcal{P}$ [natural number] $\equiv$ $c^{\$_{1}+m+1}>a^{\$_{1}+m+1}+b^{\$_{1}+m+1}$. For every $j, \mathcal{P}[j]$ from [1, Sch. 2]. 
(21) $a_{1}{ }^{m+2}-b_{1}{ }^{m+2}=\left(a_{1}{ }^{m+1}+b_{1}{ }^{m+1}\right) \cdot\left(a_{1}-b_{1}\right)+a_{1} \cdot b_{1} \cdot\left(a_{1}{ }^{m}-b_{1}{ }^{m}\right)$.

(22) $a_{1}{ }^{m+2}+b_{1}{ }^{m+2}=\left(a_{1}{ }^{m+1}-b_{1}{ }^{m+1}\right) \cdot\left(a_{1}-b_{1}\right)+a_{1} \cdot b_{1} \cdot\left(a_{1}{ }^{m}+b_{1}{ }^{m}\right)$.

(23) $\quad a^{2 \cdot m+2}-b^{2 \cdot m+2}=\frac{\left(a^{2}-b^{2}\right) \cdot\left(c \cdot\left(a^{2}+b^{2}\right)+a^{2 \cdot m}+b^{2 \cdot m}\right)}{2}$ if and only if $a^{2 \cdot m}-b^{2 \cdot m}=$ $\left(a^{2}-b^{2}\right) \cdot c$. The theorem is a consequence of (14).

(24) $a_{1}{ }^{2 \cdot m+3}+b_{1}{ }^{2 \cdot m+3}=\left(a_{1}^{2 \cdot m+2}+b_{1}^{2 \cdot m+2}\right) \cdot\left(a_{1}+b_{1}\right)-a_{1} \cdot b_{1} \cdot\left(a_{1}^{2 \cdot m+1}+\right.$ $\left.b_{1}^{2 \cdot m+1}\right)$.

(25) If $a_{1}{ }^{m}-b_{1}{ }^{m}=\left(a_{1}-b_{1}\right) \cdot k$, then $a_{1}{ }^{m+2}-b_{1}{ }^{m+2}=\left(a_{1}{ }^{m+1}+b_{1}{ }^{m+1}+\right.$ $\left.a_{1} \cdot b_{1} \cdot k\right) \cdot\left(a_{1}-b_{1}\right)$. The theorem is a consequence of $(21)$.

(26) If $a_{1}{ }^{m+2}-b_{1}{ }^{m+2}=\left(a_{1}{ }^{m+1}+b_{1}{ }^{m+1}+a_{1} \cdot b_{1} \cdot k\right) \cdot\left(a_{1}-b_{1}\right)$ and $a_{1} \cdot b_{1} \neq 0$, then $a_{1}{ }^{m}-b_{1}{ }^{m}=\left(a_{1}-b_{1}\right) \cdot k$. The theorem is a consequence of (21).

(27) If $b>0$ and $a>b$, then $\left(a^{n}-b^{n}\right) \cdot(a+b)=\left(a^{n}+b^{n}\right) \cdot(a-b)$ iff $n=1$.

(28) If $n>1$ and $b>0$ and $a>b$, then $\left(a^{n}-b^{n}\right) \cdot(a+b)>a^{n+1}-b^{n+1}$.

(29) If $n>0$ and $a>b$, then $\left(a^{n}+b^{n}\right) \cdot(a-b) \leqslant a^{n+1}-b^{n+1}$.

(30) If $p+q \mid p \cdot u+q \cdot v$, then $p+q \mid p \cdot(u+z)+q \cdot(v+z)$.

(31) $p+q \mid p \cdot(t \cdot(p+q)+z)+q \cdot z$. The theorem is a consequence of (30).

(32) If $p+q \mid u-v$, then $p+q \mid p \cdot(u+t)+q \cdot(v+t)$. The theorem is a consequence of (30).

(33) $a-b \mid a^{n}-b^{n}$.

Proof: Define $\mathcal{P}$ [natural number $] \equiv a-b \mid a^{\$_{1}}-b^{\$_{1}}$. If $\mathcal{P}[x]$, then $\mathcal{P}[x+1]$. For every $m, \mathcal{P}[m]$ from [1, Sch. 2].

(34) $a^{2}-b^{2} \mid a^{2 \cdot m}-b^{2 \cdot m}$. The theorem is a consequence of (33).

(35) $a+b \mid a^{2 \cdot m+1}+b^{2 \cdot m+1}$. The theorem is a consequence of (21), (34), and (1).

(36) $a+b \mid a^{2 \cdot m}-b^{2 \cdot m}$. The theorem is a consequence of (34).

(37) If $a+b \mid a^{n}-b^{n}$, then $a+b \mid a^{n+1}+b^{n+1}$. The theorem is a consequence of (32).

(38) (i) $a+b \mid a^{n}+b^{n}$, or

(ii) $a+b \mid a^{n}-b^{n}$.

The theorem is a consequence of (35) and (34).

(39) If $a \geqslant b$ and $c^{n}-b^{n}=a^{n}$, then $\operatorname{gcd}\left(c-b, a^{n}\right)=c-b$ and $\operatorname{gcd}\left(c-a, b^{n}\right)=$ $c-a$. The theorem is a consequence of (6) and (33).

(40) If $a$ and $b$ are relatively prime and $a+b \mid a \cdot c+b \cdot d$, then $a+b \mid c-d$. The theorem is a consequence of (32).

(41) If $a \cdot b$ and $c \cdot d$ are relatively prime, then $a$ and $c$ are relatively prime.

(42) Suppose $a>0$ and $b>0$ and $a^{n}+b^{n}=c^{n}$. Then there exists $j$ and there exists $k$ and there exists $l$ such that $j^{n}+k^{n}=l^{n}$ and $j$ and $k$ are 
relatively prime and $j$ and $l$ are relatively prime and $k$ and $l$ are relatively prime and $a=(\operatorname{gcd}(a, b)) \cdot j$ and $b=(\operatorname{gcd}(a, b)) \cdot k$ and $c=(\operatorname{gcd}(a, b)) \cdot l$.

(43) If $a>0$, then $a^{n+2}+a^{n+2} \neq b^{n+2}$. The theorem is a consequence of (42).

(44) If $x>0$ and $b<c$ and $a+b^{2}=c^{2}$, then $a+(b+x)^{2}<(c+x)^{2}$.

(45) If $q<0$ and $b<c$ and $a^{2}+b^{2}=c^{2}$, then $a^{2}+(b+q)^{2}>(c+q)^{2}$.

(46) If $x>0$ and $a^{2}+b^{2}=(b+1)^{2}$, then $a^{2}+(b-x)^{2}>(b+1-x)^{2}$. The theorem is a consequence of (45).

(47) If $a \geqslant 1$ and $(a+1)^{2}+(a+1+x)^{2} \leqslant(a+1+x+1)^{2}$, then $a^{2}+$ $(a+x)^{2}<(a+x+1)^{2}$.

(48) If $a \geqslant 1$ and $a^{2}+(a+x)^{2} \geqslant(a+x+1)^{2}$, then $(a+l+1)^{2}+(a+l+1+x)^{2}>(a+l+1+x+1)^{2}$.

Proof: Define $\mathcal{P}$ [natural number] $\equiv\left(a+\$_{1}+1\right)^{2}+\left(a+\$_{1}+1+x\right)^{2}>$ $\left(a+\$_{1}+1+x+1\right)^{2}$. $\mathcal{P}[0]$. If $\mathcal{P}[k]$, then $\mathcal{P}[k+1]$. For every $j, \mathcal{P}[j]$ from [1, Sch. 2].

(49) $a \geqslant 3$ if and only if $a^{2}+a^{2}>(a+1)^{2}$.

Proof: If $a \geqslant 3$, then $a^{2}+a^{2}>(a+1)^{2}$ by [1, (10)], [4, (81)], (48).

(50) $2^{3+m}+2^{3+m}<3^{3+m}$.

Acknowledgement: Ad Maiorem Dei Gloriam.

\section{REFERENCES}

[1] Grzegorz Bancerek. The fundamental properties of natural numbers Formalized Mathematics, 1(1):41-46, 1990.

[2] Grzegorz Bancerek. The ordinal numbers Formalized Mathematics, 1(1):91-96, 1990.

[3] E. Hatton. An intire system of Arithmetic: or, Arithmetic in all its parts. Number 6. Printed for G. Strahan, 1731. http://books . google.pl/books?id=urZJAAAMAAJ.

[4] Rafał Kwiatek. Factorial and Newton coefficients. Formalized Mathematics, 1(5):887-890, 1990.

[5] Rafał Kwiatek and Grzegorz Zwara. The divisibility of integers and integer relatively primes. Formalized Mathematics, 1(5):829-832, 1990.

[6] M.I. Mostafa. A new approach to polynomial identities The Ramanujan Journal, 8(4): 423-457, 2005. ISSN 1382-4090. do1:10.1007/s11139-005-0272-3

[7] Werner Georg Nowak. On differences of two k-th powers of integers. The Ramanujan Journal, 2(4):421-440, 1998. ISSN 1382-4090. do1 10.1023/A:1009791425210.

[8] Piotr Rudnicki and Andrzej Trybulec. Abian's fixed point theorem. Formalized Mathematics, 6(3):335-338, 1997.

[9] Michał J. Trybulec. Integers Formalized Mathematics, 1(3):501-505, 1990. 